\title{
¿Cómo tratar la hipertensión arterial sistémica? Estrategias de tratamiento actuales
}

\author{
How to treat hypertension? Current management strategies \\ Rodrigo Gopar-Nieto", Alejandro Ezquerra-Osorio²*, Nancy L Chávez-Gómez², \\ Daniel Manzur-Sandovaß ${ }^{3}$ y Grecia I. M. Raymundo-Martínez ${ }^{2}$ \\ ${ }^{1}$ Unidad Coronaria; ${ }^{2}$ Servicio de Cardiología; ${ }^{3}$ Departamento de Terapia Intensiva Cardiovascular. Instituto Nacional de Cardiología Ignacio Chávez, \\ Ciudad de México, México
}

\begin{abstract}
Resumen
La hipertensión arterial sistémica es una enfermedad que afecta casi a la mitad de la población. Su compleja fisiopatología, que afecta principalmente a los sistemas renal, hormonal, cardiovascular y neurológico, ha permitido tener diferentes estrategias farmacológicas para tratar cada uno de esos sistemas y así regular la tensión arterial. La American Heart Association en el 2017, la European Society of Cardiology en el 2018 y, por último, la International Society of Hypertension en el 2020, publicaron recomendaciones para el diagnóstico, monitoreo y tratamiento de la hipertensión arterial. La definición de tensión arterial normal o hipertensión varía de acuerdo con cada guía. Las recomendaciones en los cambios del estilo de vida son muy similares, al igual que el tratamiento farmacológico mediante inhibidores del sistema renina-angiotensina, antagonistas de canales de calcio y diuréticos tiazídicos y solo en casos seleccionados el uso de antagonistas de la aldosterona o betabloqueadores.
\end{abstract}

Palabras clave: Hipertensión arterial. Estrategias de tratamiento. Guías de tratamiento.

\begin{abstract}
Hypertension is a disease that affects almost half of the population. Its complex pathophysiology, mainly affecting the renal, hormonal, cardiovascular and neurological systems, has allowed us to have different pharmacological strategies to treat each of these systems and thus regulate blood pressure. The American Heart Association in 2017, the European Society of Cardiology in 2018, and the American Society of Hypertension in 2020 published their recommendations for the diagnosis, monitoring and treatment of arterial hypertension. The definition of normal blood pressure or hypertension varies according to each guideline. Recommendations on lifestyle and pharmacological therapy are very similar in the guidelines. They recommend blockers of the renin-angiotensin system, calcium antagonists and thiazides, and only in selected cases the use of mineralocorticoid receptor antagonist or beta-blockers.
\end{abstract}

Key words: Hypertension. Treatment strategies. Guidelines.

Correspondencia:

*Alejandro Ezquerra-Osorio

E-mail: aeo900@yahoo.com.mx
Disponible en internet: 02-12-2020 Arch Cardiol Mex. 2021;91(4):493-499 www.archivoscardiologia.com

( C) 2020 Instituto Nacional de Cardiología Ignacio Chávez. Publicado por Permanyer. Este es un artículo open access bajo la licencia CC BY-NC-ND (http://creativecommons.org/licenses/by-nc-nd/4.0/).

Fecha de recepción: 21-06-2020 Fecha de aceptación: 06-11-2020 DOI: $10.24875 /$ ACM. 200003011 


\section{Introducción}

La hipertensión arterial sistémica es una de las enfermedades con mayor prevalencia en la población mundial, ya que aparece en el $30-45 \%$ de los adultos. Afecta a todos los sistemas del organismo humano, por lo que un control poco adecuado puede tener múltiples manifestaciones clínicas. Debido a la gran diversidad de información, varias asociaciones han publicado sus recomendaciones y en esta revisión mostraremos las generalidades de esta enfermedad y compararemos las estrategias de manejo disponibles.

\section{Fisiopatología}

La hipertensión arterial se origina por una combinación de factores genéticos y ambientales, de los cuales la herencia constituye del 30 al $50 \%$, resultando la mayoría de los casos poligénicos, mientras que los factores ambientales inducen modificaciones epigenéticas. El mantenimiento de la presión arterial normal es el resultado del equilibrio entre el gasto cardiaco y las resistencias vasculares periféricas. Esto se puede afectar por alteraciones en el sistema renal, hormonal, cardiovascular y neurológico, las cuales pueden elevar el gasto cardiaco, resistencias o ambas, ocasionando hipertensión $n^{1,2}$.

Los principales mecanismos fisiopatológicos para la aparición de hipertensión arterial sistémica son: a) la disminución en excreción renal de sodio, que aumenta el volumen y precarga; b) la activación de endotelina 1 , que ocasiona vasoconstricción sistémica ${ }^{3}$; c) a nivel vascular la disfunción endotelial por especies reactivas de oxígeno evita la liberación de óxido nitroso, un potente vasodilatador, y d) la hiperactividad simpática, tanto central y periférica, que aumenta las resistencias periféricas. Por otra parte, recientemente se ha demostrado que la obesidad es un factor estimulante de la actividad simpática y que hay ciertas enfermedades, como la artritis reumatoide, que aumentan el riesgo de padecerla. También se debe tener en cuenta que la hipertensión arterial se puede asociar al consumo de ciertos fármacos, como inhibidores de calcineurina (tacrolimús y ciclosporina) y anticuerpos monoclonales inhibidores de factor de crecimiento vascular endotelial (bevacizumab), ya que disminuyen la producción de óxido nítrico, aumentan la endotelina 1 y alteran de la natriuresis, principalmente en pacientes mayores de 60 años, siendo reversible al descontinuar el medicamento ${ }^{4}$. Por consiguiente, al entender que la hipertensión es resultado de varios mecanismos, se justifica iniciar tratamiento con combinaciones de estrategias y fármacos.

\section{Modificaciones en el estilo de vida}

En primer lugar se encuentran las estrategias dietéticas, que incluyen la dieta mediterránea y la dieta DASH (Dietary Approaches to Stop Hypertension), las cuales demostraron disminución de eventos cardiovasculares mayores y descensos medibles de la tensión arterial sistólica ${ }^{5,6}$.

El consumo de sodio ha sido motivo de controversia, ya que en los estudios epidemiológicos se ha encontrado que tanto la ingesta de niveles bajos como altos se asocia a eventos cardiovasculares adversos ${ }^{7}$. La European Society of Cardiology (ESC) propone un consumo de sal no mayor de $5 \mathrm{~g} / \mathrm{d}$ ía, es decir, lo equivalente a $2 \mathrm{~g}$ de sodio, mientras que la International Society of Hypertension (ISH) recomienda el consumir $4.7 \mathrm{~g} / \mathrm{día}$ de este elemento ${ }^{8,9}$. Por último, debemos dejar en claro que el objetivo de las recomendaciones es evitar añadir sal a los alimentos, además de establecer estrategias sociales para disminuir o restringir su uso.

El consumo de alcohol se ha asociado con la aparición de desenlaces cardiovasculares adversos, sobre todo con el consumo excesivo de estas bebidas, debido a su efecto presor. Las recomendaciones actuales limitan su consumo a 14 unidades/semana en hombres y 8 unidades/semana en mujeres, teniendo en cuenta que 1 unidad es equivalente a $125 \mathrm{ml}$ de vino o $250 \mathrm{ml}$ de cerveza ${ }^{8,10}$. En cuanto a otras bebidas, debemos resaltar los efectos benéficos del consumo del café y del té negro o verde, ya que se ha demostrado que pueden disminuir la tensión arterial, aunque de manera discreta. Por último, hay que desaconsejar el consumo de bebidas endulzadas debido a su alto contenido en sodio y a su relación con la aparición de sobrepeso, obesidad, síndrome metabólico y diabetes mellitus ${ }^{11}$.

En cuanto al sobrepeso y la obesidad se ha encontrado que un índice de masa corporal $\geq 25 \mathrm{~kg} / \mathrm{m}^{2}$ aumenta el riesgo de elevación de la tensión arterial en un $50 \%$, además de tener una asociación con mayor mortalidad cardiovascular. Para reducir este riesgo se debe incentivar la pérdida de peso y para eso se debe establecer un programa multidisciplinario que incluya modificación dietética, ejercicio regular, asesorías motivacionales y, en casos seleccionados, intervenciones bariátricas. Todas las estrategias deben encaminarse a tener un índice de masa corporal entre 20 y $25 \mathrm{~kg} / \mathrm{m}^{2}$, además de una circunferencia de cintura $<94 \mathrm{~cm}$ en hombres $y<80 \mathrm{~cm}$ en mujeres ${ }^{12}$. 
La actividad física aeróbica disminuye hasta $8.3 \mathrm{mmHg}$ la tensión arterial sistólica y $5.2 \mathrm{mmHg}$ la diastólica en pacientes con hipertensión arterial. Se recomienda realizar al menos 30 minutos de actividad aeróbica de intensidad moderada durante 5 a 7 días a la semana, mientras que el aumentar la actividad física moderada a 300 minutos/semana o la actividad vigorosa a 150 minutos/semana pueden mejorar en mayor proporción los niveles de tensión arterial ${ }^{13}$.

Por último, hay que desalentar el consumo de tabaco mediante el consejo médico, estrategias farmacológicas con vareniclina y terapia de reemplazo con nicotina o bupropión además de consejería conductual, las cuales pueden tener una tasa de éxito del 70 al $100 \%{ }^{14}$.

\section{Opciones farmacológicas}

A pesar de lo anteriormente mencionado, en la gran mayoría de los pacientes los cambios en el estilo de vida no son suficientes, por lo que se debe añadir terapia farmacológica. Las principales clases de fármacos que pueden utilizarse son: inhibidores del sistema renina-angiotensina, antagonistas de canales de calcio y diuréticos tiazídicos. En la siguiente sección desarrollaremos sus principales características y efectos adversos, mientras que en la tabla 1 se muestran los principales fármacos utilizados.

\section{Bloqueadores del sistema renina- angiotensina}

En este apartado se encuentran los inhibidores de la enzima convertidora de angiotensina (IECA) y los antagonistas del receptor de angiotensina (ARA II), los cuales tienen una eficacia similar en cuanto a la disminución de albuminuria tanto en pacientes diabéticos como en los no diabéticos, la regresión de la hipertrofia ventricular izquierda y mejoría de la función ventricular izquierda, junto con reducción en la incidencia de fibrilación atrial ${ }^{15,16}$. Debemos enfatizar que el administrar un IECA y un ARA, o combinarlos con aliskiren, no proporciona ningún beneficio, causando únicamente mayor incidencia de lesión renal aguda e hiperpotasemia, por lo que estas combinaciones se encuentran contraindicadas ${ }^{17}$. Además, hay que prestar atención a que este tipo de medicamentos están prohibidos durante el embarazo debido al riesgo de agenesia renal y otros defectos congénitos (en el primer trimestre, malformaciones del sistema nervioso central y cardia$\mathrm{co}$; en el segundo trimestre, malformaciones urogenitales y renales).
Dentro de los efectos adversos más comunes se encuentra la tos, principalmente con Ios IECA, y en este caso se deberá hacer cambio a un ARA. Ambos tipos de medicamentos pueden ocasionar un ligero incremento en la creatinina, sin embargo, deben descontinuarse a menos que sea mayor al 30\% de los niveles basales durante las primeras tres semanas. En muy pocos casos se puede presentar acidosis tubular renal tipo $4^{8,9}$.

\section{Antagonistas de canales de calcio}

Una de las grandes ventajas es que no requieren monitorización por medio de estudios de laboratorio, además de ser antianginosos y antiarrítmicos. Su mecanismo de acción se centra en el bloqueo de la apertura de los canales dependientes de voltaje en los cardiomiocitos y en el músculo liso vascular, lo que disminuye la tensión arterial por vasodilatación arterial periférica. Su efectividad es comparable a la de los IECA y los diuréticos tiazídicos, con la ventaja de mayor prevención de eventos vasculares cerebrales isquémicos, además de una relación favorable en cuanto al costo-beneficio ${ }^{18}$. Su principal efecto adverso es el edema de miembros inferiores, el cual es dependiente de la dosis, y puede mejorar con la administración concomitante de IECA o ARA. Otros efectos adversos son la hiperplasia gingival y la aparición de bloqueos en el sistema de conducción.

\section{Diuréticos tiazídicos}

Esta clase constituye una de las piedras angulares y tiene como ventaja la prevención de la aparición de insuficiencia cardiaca. Existe un gran debate en cuanto al tipo de diurético tiazídico ideal, ya que se han encontrado grandes beneficios con la administración de indapamida y clortalidona, sin embargo, nunca se ha hecho una comparación directa entre estos y los medicamentos clásicos, hidroclorotiazida y bendrofluorazida $^{19}$. Cabe resaltar que son muy utilizados debido a su sinergia con el resto de clases farmacológicas para el tratamiento de la hipertensión. Su mecanismo de acción es el bloqueo del cotransportador de $\mathrm{NaCl}$ en el túbulo contorneado distal. Dentro de sus efectos adversos podemos encontrar la intolerancia a la glucosa, hipopotasemia, hipomagnesemia e hiponatremia, además de ataques de gota, elevación de los lípidos séricos, hipotensión ortostática en pacientes geriátricos, alcalosis metabólica (por disminución en la excreción urinaria de calcio) y disfunción eréctil. 
Tabla 1. Principales fármacos para el manejo de la hipertensión arterial sistémica

\begin{tabular}{|c|c|c|c|}
\hline Clase & Fármaco & Dosis usual (mg/día) & Frecuencia \\
\hline \multicolumn{4}{|c|}{ Agentes de primera línea } \\
\hline Tiazidas & $\begin{array}{l}\text { Clortalidona } \\
\text { Hidroclorotiazida }\end{array}$ & $\begin{array}{l}12.5-25 \\
25-50\end{array}$ & $\begin{array}{l}1 \\
1\end{array}$ \\
\hline Inhibidores de la enzima convertidora de angiotensina & $\begin{array}{l}\text { Captopril } \\
\text { Enalapril } \\
\text { Lisinopril } \\
\text { Perindopril } \\
\text { Ramipril }\end{array}$ & $\begin{array}{c}12.5-150 \\
5-40 \\
10-40 \\
4-16 \\
2.5-20\end{array}$ & $\begin{array}{c}2-3 \\
1-2 \\
1 \\
1 \\
1-2\end{array}$ \\
\hline Antagonistas de los receptores de angiotensina II & $\begin{array}{l}\text { Azilsartán } \\
\text { Candesartán } \\
\text { Irbesartán } \\
\text { Losartán } \\
\text { Olmesartán } \\
\text { Telmisartán } \\
\text { Valsartán }\end{array}$ & $\begin{array}{c}40-80 \\
8-32 \\
150-300 \\
50-100 \\
20-40 \\
20-80 \\
80-320\end{array}$ & $\begin{array}{c}1 \\
1 \\
1 \\
1-2 \\
1 \\
1 \\
1\end{array}$ \\
\hline Antagonistas de canales de calcio dihidropiridínicos & $\begin{array}{l}\text { Amlodipino } \\
\text { Nifedipino }\end{array}$ & $\begin{array}{l}2.5-10 \\
30-90\end{array}$ & $\begin{array}{l}1 \\
1\end{array}$ \\
\hline \multicolumn{4}{|c|}{ Agentes de segunda línea } \\
\hline Diuréticos de asa & $\begin{array}{l}\text { Bumetanida } \\
\text { Furosemida }\end{array}$ & $\begin{array}{l}0.5-2 \\
20-80\end{array}$ & $\begin{array}{l}2 \\
2\end{array}$ \\
\hline Diuréticos antagonistas de receptores de aldosterona & $\begin{array}{l}\text { Eplerenona } \\
\text { Espironolactona }\end{array}$ & $\begin{array}{l}50-100 \\
25-100\end{array}$ & $\begin{array}{c}1-2 \\
2\end{array}$ \\
\hline Betabloqueadores cardioselectivos & $\begin{array}{l}\text { Atenolol } \\
\text { Bisoprolol } \\
\text { Metoprolol tartrato } \\
\text { Metoprolol succinato }\end{array}$ & $\begin{array}{c}25-100 \\
2.5-10 \\
100-200 \\
50-200\end{array}$ & $\begin{array}{l}2 \\
1 \\
2 \\
1\end{array}$ \\
\hline Betabloqueadores cardioselectivos y vasodilatadores & Nevibolol & $5-40$ & 1 \\
\hline Betabloqueadores no cardioselectivos & $\begin{array}{l}\text { Nadolol } \\
\text { Propranolol (larga acción) }\end{array}$ & $\begin{array}{l}40-120 \\
80-160\end{array}$ & $\begin{array}{l}1 \\
1\end{array}$ \\
\hline Betabloqueadores combinados & $\begin{array}{l}\text { Carvedilol } \\
\text { Carvedilol fosfato } \\
\text { Labetalol }\end{array}$ & $\begin{array}{c}12.5-50 \\
20-80 \\
200-800\end{array}$ & $\begin{array}{l}2 \\
1 \\
2\end{array}$ \\
\hline Inhibidores directos de la renina & Aliskiren & $150-300$ & 1 \\
\hline Alfabloqueantes & $\begin{array}{l}\text { Doxazosina } \\
\text { Prazosina } \\
\text { Terazosina }\end{array}$ & $\begin{array}{l}1-16 \\
2-20 \\
1-20\end{array}$ & $\begin{array}{c}1 \\
2-3 \\
1-2\end{array}$ \\
\hline Agonistas alfa 2 y otros fármacos de acción central & Metildopa & $250-1000$ & 2 \\
\hline Vasodilatadores directos & $\begin{array}{l}\text { Hidralazina } \\
\text { Minoxidil }\end{array}$ & $\begin{array}{c}100-200 \\
5-100\end{array}$ & $\begin{array}{l}2-3 \\
1-3\end{array}$ \\
\hline
\end{tabular}

\section{Betabloqueadores}

Han demostrado disminuir la aparición de insuficiencia cardiaca y evento vascular cerebral isquémico, con mayor beneficio en personas con infarto de miocardio, además de ser adecuados para el control de la frecuencia cardiaca y la angina crónica estable. También se pueden utilizar en embarazadas o mujeres en edad reproductiva. Dentro de los efectos adversos se encuentran el broncoespasmo y el retardo en la conducción cardiaca ${ }^{8,9}$.

\section{Otras clases farmacológicas}

Los antagonistas de la aldosterona pueden ser un complemento muy útil, sobre todo en casos de difícil 
Tabla 2. Comparación entre los lineamientos para el manejo de la hipertensión arterial

\begin{tabular}{|c|c|c|c|c|}
\hline & АНА 2017 & ESC 2018 & ISH 2020 & SIAC 2020 \\
\hline Normal & $<120 \mathrm{y}<80 \mathrm{mmHg}$ & $120-129 \mathrm{y} / 0$ 80-84 mmHg & $<130 \mathrm{y}<85 \mathrm{mmHg}$ & $120-129 \mathrm{y} / 0$ 80-84 mmHg \\
\hline Prehipertensión & $\begin{array}{l}\text { Elevada: } 120-129 \text { y } \\
<80 \mathrm{mmHg}\end{array}$ & $\begin{array}{l}\text { Normal elevada: } 130-139 \\
\text { y/o } 85-89 \mathrm{mmHg}\end{array}$ & $\begin{array}{l}\text { Normal elevada: } 130-139 \\
\text { y/o } 85-89 \mathrm{mmHg}\end{array}$ & $\begin{array}{l}\text { Normal elevada: } 130-139 \\
\text { y/o } 85-89 \mathrm{mmHg}\end{array}$ \\
\hline Clasificación & $\begin{array}{l}\text { Estadio } 1: 130-139 \text { o } \\
80 \text { - } 89 \mathrm{mmHg} \\
\text { Estadio } 2: \\
\geq 140 \text { o } 90 \mathrm{mmHg} \\
\text { Crisis hipertensiva: } \\
\geq 180 \text { o } 120 \mathrm{mmHg}\end{array}$ & $\begin{array}{l}\text { Grado 1: } 140-159 \text { y/o 90-99 } \\
\text { mmHg } \\
\text { Grado 22: } 160-179 \text { y/o 100-109 } \\
\text { mmHg } \\
\text { Grado 3: } \geq 180 \text { y/o } \geq 110 \mathrm{mmHg} \\
\text { Hipertensión sistólica aislada: } \\
\geq 140 \text { y }<90 \mathrm{mmHg}\end{array}$ & $\begin{array}{l}\text { Grado } 1: 140-159 \\
\text { y/o } 90-99 \mathrm{mmHg} \\
\text { Grado } 2: \geq 160 \\
\text { y/o } \geq 100 \mathrm{mmHg}\end{array}$ & $\begin{array}{l}\text { Grado 1: } 140-159 \text { y/o } 90-99 \\
\text { mmHg } \\
\text { Grado 22: } 160-179 \text { y/o } \\
\text { 100-109 mmHg } \\
\text { Grado } 3: \geq 180 \\
\text { y/o } \geq 110 \mathrm{mmHg} \\
\text { Hipertensión sistólica } \\
\text { aislada: } \geq 140 \text { y }<90 \mathrm{mmHg}\end{array}$ \\
\hline Estilo de vida & Siempre & $\begin{array}{l}\text { Siempre, tratamiento inicial en } \\
\text { normal elevada y grado } 1 \text { (si no } \\
\text { hay riesgo cardiovascular } \\
\text { elevado o daño a órgano } \\
\text { blanco) }\end{array}$ & $\begin{array}{l}\text { Siempre, tratamiento inicial } \\
\text { en normal elevada y grado } 1 \\
\text { (si no hay riesgo } \\
\text { cardiovascular elevado, } \\
\text { enfermedad renal, diabetes } \\
\text { mellitus, enfermedad } \\
\text { cardiovascular o daño a } \\
\text { órgano blanco) }\end{array}$ & $\begin{array}{l}\text { Siempre, tratamiento } \\
\text { inicial en normal elevada } \\
\text { y grado } 1 \text { (si no hay riesgo } \\
\text { cardiovascular elevado o } \\
\text { daño a órgano blanco) }\end{array}$ \\
\hline $\begin{array}{l}\text { ¿Cuándo iniciar } \\
\text { tratamiento } \\
\text { farmacológico? }\end{array}$ & $\begin{array}{l}\text { Estadio 1: } \\
\text { ASCVD } \geq 10 \% \\
\text { Estadio 2: siempre }\end{array}$ & $\begin{array}{l}\text { Grado 1: en alto riesgo } \\
\text { cardiovascular, daño órgano o } \\
\text { no llegar a meta 3-6 meses con } \\
\text { cambio en estilo de vida } \\
\text { Grado } 2 \text { y 3: siempre }\end{array}$ & $\begin{array}{l}\text { Grado 1: en alto elevado } \\
\text { riesgo enfermedad renal, } \\
\text { diabetes mellitus, } \\
\text { enfermedad cardiovascular } \\
\text { o daño a órgano blanco) } \\
\text { cardiovascular, } \\
\text { comorbilidades o no llegar a } \\
\text { meta 3-6 meses con cambio } \\
\text { en estilo de vida } \\
\text { Grado 2: siempre }\end{array}$ & $\begin{array}{l}\text { Grado 1: en alto riesgo } \\
\text { cardiovascular, daño } \\
\text { órgano o no llegar a meta } \\
3-6 \text { meses con cambio en } \\
\text { estilo de vida } \\
\text { Grado } 2 \text { y } 3 \text { : siempre }\end{array}$ \\
\hline $\begin{array}{l}\text { ¿Qué } \\
\text { antihipertensivos } \\
\text { son elección? }\end{array}$ & $\begin{array}{l}\text { Elección de acuerdo } \\
\text { con comorbilidades, } \\
\text { combinar diferentes } \\
\text { familias }\end{array}$ & $\begin{array}{l}\text { Elección de acuerdo con } \\
\text { comorbilidades o con iniciar } \\
\text { IECA/ARA + calcio antagonista } \\
\text { o diurético }\end{array}$ & $\begin{array}{l}\text { Iniciar IECA/ARA + calcio } \\
\text { antagonista, } \\
\text { diurético el } 3 .^{\circ} \text { en agregar }\end{array}$ & $\begin{array}{l}\text { Elección de acuerdo con } \\
\text { comorbilidades o con } \\
\text { iniciar IECA/ARA + calcio } \\
\text { antagonista o diurético }\end{array}$ \\
\hline Monoterapia & $\begin{array}{l}\text { Estadio } 1 \mathrm{y} \\
\text { ASCVD }<10 \%\end{array}$ & $\begin{array}{l}\text { Grado } 1 \text { bajo riesgo } \\
\text { cardiovascular, adultos frágiles } \\
y \geq 80 \text { años }\end{array}$ & $\begin{array}{l}\text { Grado } 1 \text { de bajo riesgo } \\
\text { cardiovascular, adultos } \\
\text { frágiles } y \geq 80 \text { años }\end{array}$ & $\begin{array}{l}\text { Grado } 1 \text { bajo riesgo } \\
\text { cardiovascular, adultos } \\
\text { frágiles } y \geq 80 \text { años }\end{array}$ \\
\hline $\begin{array}{l}\text { Hipertensión } \\
\text { resistente }\end{array}$ & $\begin{array}{l}\text { Maximizar diurético, } \\
\text { agregar } \\
\text { espironolactona u } \\
\text { otro mecanismo de } \\
\text { acción }\end{array}$ & $\begin{array}{l}\text { Espironolactona } 12.5 \mathrm{a} 50 \mathrm{mg} \\
\text { (contraindicado en TFG } \\
\leq 45 \mathrm{ml} / \mathrm{min} \text { o } \mathrm{K} \geq 4.5 \mathrm{mEq} \text { ) }\end{array}$ & $\begin{array}{l}\text { Espironolactona } 12.5 \text { a } 50 \\
\mathrm{mg} \text { (contraindicado en } \\
\mathrm{TFG} \leq 45 \mathrm{ml} / \mathrm{min} \\
\mathrm{o} \mathrm{K} \geq 4.5 \mathrm{mEq} \text { ) }\end{array}$ & $\begin{array}{l}\text { Espironolactona } 12.5 \mathrm{mg} \text { a } \\
50 \mathrm{mg} \text { (contraindicado en } \\
\mathrm{TFG} \leq 45 \mathrm{ml} / \mathrm{min} 0 \\
\mathrm{~K} \geq 4.5 \mathrm{mEq} \text { ) }\end{array}$ \\
\hline Metas & $\begin{array}{l}\text { Todos }<130 / 80 \\
\text { mmHg, no aclara } \\
\text { meta diastólica en } \\
\geq 65 \text { años }\end{array}$ & $\begin{array}{l}3 \text { meses para llegar a meta } \\
\text { Al menos } 130 / 80 \mathrm{mmHg} \text {, } \\
<65 \text { años: idealmente } \\
120-129 /<80 \mathrm{mmHg} \\
\geq 65 \text { años: entre } \\
130-140 \text { y } 80 \mathrm{mmHg}\end{array}$ & $\begin{array}{l}3 \text { meses para llegar a meta } \\
\text { < } 65 \text { años: } \\
<120-130 / 70-80 \mathrm{mmHg} \\
\geq 65 \text { años: } \\
<140 / 90 \mathrm{mmHg} \text { e } \\
\text { individualizar }\end{array}$ & $\begin{array}{l}\text { 3-6 meses para llegar a } \\
\text { meta } \\
\text { Al menos } 130 / 80 \mathrm{mmHg} \text {, } \\
<65 \text { años: idealmente } \\
120-129 /<80 \mathrm{mmHg} \\
\geq 65 \text { años: entre } \\
130-140 \text { y } 80 \mathrm{mmHg}\end{array}$ \\
\hline Lactancia & No hace mención & Evitar propanolol y nifedipino & $\begin{array}{l}\text { Evitar atenolol, propanolol y } \\
\text { nifedipino }\end{array}$ & No hace mención \\
\hline
\end{tabular}

AHA: American Heart Association; ESC: European Society of Cardiology, ISH: International Society of Hypertension; SIAC: Sociedad Interamericana de Cardiología; ASCVD: atherosclerotic cardiovascular disease; IECA: inhibidores de la enzima convertidora de angiotensina; ARA: inhibidores del receptor de angiotensina; TFG: tasa de filtración glomerular.

control. Se ha demostrado que añadir espironolactona, en casos de hipertensión resistente, es superior a doxazosina y bisoprolol ${ }^{20}$. Hay que mencionar que la eplerenona tiene menos efectos sexuales (tales como ginecomastia, disfunción eréctil o sangrado uterino) que la espironolactona. En cuanto a otra clase farmacológica, 
los bloqueadores alfa pueden requerirse en la hiperplasia prostática y enfermedad renal crónica. Por último, los vasodilatadores directos, principalmente la hidralazina, pueden utilizarse en hipertensión severa durante el embarazo o en preeclampsia ${ }^{8,9}$.

\section{Comparación entre las recomendaciones}

Los valores para el diagnóstico, tratamiento y control de presión arterial han tenido cambios y controversias en los últimos tres años de acuerdo con lo publicado con la guía 2017 de la American Heart Association $(\mathrm{AHA})^{9}$, la guía 2018 de la $\mathrm{ESC}^{8}$, la ISH que publicó sus lineamientos en mayo del $2020^{21}$, y la Sociedad Interamericana de Cardiología (SIAC) en julio de $2020^{22}$.

Se ha comentado que existen grandes diferencias entre ellas, pero la realidad es que tienen bastantes similitudes. Todas dan más peso a la confirmación o diagnóstico ambulatorio, medición con técnica correcta y estimación del riesgo cardiovascular, a pesar de utilizar diferentes escalas para su medición: ASCVD (atherosclerotic cardiovascular disease) para las guías americanas y SCORE (Systematic Coronary Risk EvaIuation) para las europeas y el consenso de la SIAC,8,9,22. Asimismo, concuerdan en que todos deben tener cambios en estilo de vida, además de que se debe preferir la combinación de medicamentos en una sola pastilla, utilizar monoterapia solo para ciertos casos y se reservan los betabloqueadores para condiciones específicas como la cardiopatía isquémica, arritmias y los síndromes aórticos ${ }^{23}$. En cuanto a los medicamentos prohibidos en la lactancia, concuerdan en no prescribir propanolol y nifedipino ${ }^{8,21}$.

En donde existen ciertas diferencias es en la definición de la tensión arterial normal, ya que la guía 2017 la define con $<120 / 80 \mathrm{mmHg}$, mientras que la 2018 con $<120$ $129 / 80-84 \mathrm{mmHg}$ y la de 2020 con $<130 / 85 \mathrm{mmHg}$. Por otro lado, las cuatro guías mencionan que se puede dar monoterapia en el estadio 1 de acuerdo con el riesgo cardiovascular; sin embargo, hay diferencias entre guías para definir dicho estadio: para la 2017 el corte es 130139/80-89 mmHg, mientras que la 2018 y 2020 concuerdan con 140-159/90-99 mmHg $8,9,21,22$.

Otra disparidad es la meta de tensión arterial. En la guía 2017 se menciona que todos deben tener menos de $130 / 80 \mathrm{mmHg}$ y solo los mayores de 65 años no tienen meta diastólica ${ }^{8,9}$. Los europeos proponen al menos $130 / 80 \mathrm{mmHg}$ y en menores de 65 años idealmente $120-129 /<80 \mathrm{mmHg}$. Las guías 2018 y 2020 proponen en mayores de 65 años entre $130-140 / 80 \mathrm{mmHg}$ $y<140 / 90 \mathrm{mmHg}$, respectivamente $\mathrm{e}^{8,9,21}$.
Independientemente de cómo definamos hipertensión o qué guía se utilice, no olvidemos que tratamos pacientes y no solo valores de presión arterial, por lo cual siempre debemos individualizar el tratamiento. Para comparar y contrastar las recomendaciones recomendamos revisar la tabla 2.

\section{Conclusiones}

La hipertensión arterial es altamente prevalente, por lo que se necesita un conocimiento a fondo de sus mecanismos fisiopatológicos para poder establecer una adecuada estrategia de control. Es importante que el paciente comprenda que el tratamiento de hipertensión arterial actualmente se basa en recomendaciones con cambio en el estilo de vida y estrategias farmacológicas y no solo se basa en medicinas. Un buen apego a los hábitos en la vida diaria nos permitirá reducir la dosis de medicamentos y así evitar efectos secundarios. A pesar de existir muchos lineamientos disponibles, tenemos que hacer énfasis en que debemos guiarnos por metas para poder lograr un adecuado control y evitar complicaciones.

\section{Financiación}

Ninguno.

\section{Conflictos de intereses}

Los autores declaran no tener conflicto de intereses.

\section{Responsabilidades éticas}

Protección de personas y animales. Los autores declaran que para esta investigación no se han realizado experimentos en seres humanos ni en animales.

Confidencialidad de los datos. Los autores declaran que en este artículo no aparecen datos de pacientes.

Derecho a la privacidad y consentimiento informado. Los autores declaran que en este artículo no aparecen datos de pacientes.

\section{Bibliografía}

\footnotetext{
1. Singh $M$, Mensah GA, Bakris G. Pathogenesis and clinical physiology of hypertension. Cardiol Clin. 2010;28:545-59.

2. Carey RM, Munter P, Bosworth HB, Whelton P. Prevention and Control of Hypertension: JACC Health Promotion Series. J Am Coll Cardiol. 2018;72:1278-93.
} 
3. Taddei S, Bruno RS, Masi S, Solini A. Epidemiology and pathophysiology of hypertension. En: Camm AJ, Luscher TF, Maurer G, Serruys PW. ESC CardioMed, 3th ed. Oxford University Press; 2020. pp. 14.

4. Tarun S, Azeema OA, Manjari S. Pathophysiology of essential hypertension: an update. Expert Rev Cardiovasc Ther. 2018;16(12):879-87.

5. Estruch R, Ros E, Salas-Salvadó J, Covas MI, Corella D, Arós F, et al Primary prevention of cardiovascular diseases with a mediterranean diet supplemented with extra-virgin olive oil or nuts. $\mathrm{N}$ Engl $\mathrm{J}$ Med. 2018;378:e34.

6. Appel LJ, Moore TJ, Obarzanek E, Vollmer WM, Svetkey LP, Sacks FM, et al. A clinical trial of the effects of dietary patterns on blood pressure. N Engl J Med. 1997;336:1117-24.

7. Grillo A, Salvi L, Coruzzi P, Salvi P, Parati G. Sodium intake and hypertension. Nutrients. 2019;11:1970.

8. Williams B, Mancia G, Spiering W, Rosei EA, Azizi M, Burnier M, et al. 2018 ESC/ESH Guidelines for the Management of Arterial Hypertension. Eur Heart J. 2018;39(33):3021-310.

9. Whelton PK, Carey RM, Aronow WS, Casey DE, Collins KJ, Himmelfarb CD, et al. 2017 ACC/AHA/AAPA/ABC/ACPM/AGS/APhA/ ASH/ASPC/NMA/PCNA Guideline for the Prevention, Detection, Evaluation, and Management of High Blood Pressure in Adults: A Report of the American College of Cardiology/American Heart Association Task Force on Clinical Practice Guidelines. Hypertension. 2017;71:e13-e115.

10. Puddey IB, Mori TA, Barden AE, Beilin LJ. Alcohol and hypertension-new insights and lingering controversies. Curr Hypertens Rep. 2019;21:79.

11. Liu Q, Ayoub-Charette S, Ahmad Khan T, Au-Yeng F, Blanco Mejia S, De Souza RJ, et al. Important food sources of fructose-containing sugars and incident hypertension: a systematic review and dose-response meta-analysis of prospective cohort studies. J Am Heart Assoc. 2019;8:e010977.

12. Neter JE, Stam BE, Kok FJ, Grobbee DE, Geleijnse JM. Influence of weight reduction on blood pressure: a meta-analysis of randomized controlled trials. Hypertension. 2003;42:878-84.

13. Rossi A, Dikareva A, Bacon SL, Daskalopoulou SS. The impact of physical activity on mortality in patients with high blood pressure: a systematic review. J Hypertens. 2012;30:1277-88.
14. Cahill K, Stevens S, Perera R, Lancaster T. Pharmacological interventions for smoking cessation: an overview and network meta-analysis. Cochrane Database Syst Rev. 2013;5:CD009329.

15. Yusuf S, Teo KK, Pogue J, Dyal L, Copland I, Schumacher $\mathrm{H}$, et al. Telmisartan, ramipril, or both in patients at high risk for vascular events. N Engl J Med. 2008;358:1547-59.

16. Wright JT, Bakris G, Greene T, Agodoa LY, Appel LJ, Charleston J, et al. Effect of blood pressure lowering and antihypertensive drug class on progression of hypertensive kidney disease: results from the AASK trial. JAMA. 2002;288:2421-31.

17. Parving HH, Brenner BM, McMurray JJ, de Zeeuw D, Haffner SM, Solomon SD, et al. Cardiorenal end points in a trial of aliskiren for type 2 diabetes. N Engl J Med. 2012;367:2204-13.

18. ALLHAT officers and coordinators. Major outcomes in high-risk hypertensive patients randomized to angiotensin-converting enzyme inhibitor or calcium channel blocker vs diuretic: The Antihypertensive and Lipid-Lowering Treatment to Prevent Heart Attack Trial (ALLHAT). JAMA. 2002;288:2981-97.

19. Wright TJ, Dunn JK, Cutler JA, Davis BR, Cushman WC, Ford CE, et al. Outcomes in hypertensive black and nonblack patients treated with chlorthalidone, amlodipine, and Lisinopril. JAMA 2005;293:1595-608.

20. Williams B, MacDonald TM, Morant S, Webb DJ, Sever P, Mclnnes G, et al. Spironolactone versus placebo, bisoprolol, and doxazocin to determine the optimal treatment for drug-resistant hypertension (PATHWAY-2): a randomized, double-blind, crossover trial. Lancet. 2015;386:2059-68.

21. Unger T, Borghi C, Charchar F, Khan NA, Poulter NR, Prabhakaran D, et al. 2020 International Society of Hypertension global hypertension practice guidelines. J Hypertens. 2020;38:982-1004.

22. Wyss F, Coca A, López-Jaramillo P, Ponte-Negretti C. Position statement of the Interamerican Society of Cardiology (IASC) on the current guidelines for the prevention, diagnosis and treatment of arterial hypertension 20172020. International Journal of Cardiology: Hypertension. 2020;7:1000061.

23. Whelton PK, Williams B. The 2018 European Society of Cardiology/European Society of Hypertension and 2017 American College of Cardiology/American Heart Association Blood Pressure Guidelines. More Similar Than Different. JAMA. 2018;320(17):1749-50. 